\title{
Entre école obligatoire et formation postobligatoire. Une approche socioculturelle de la trajectoire scolaire pour comprendre les dynamiques d'accrochage scolaire Between compulsory schooling and post-compulsory training. A sociocultural approach to the educational trajectory in to understand the dynamics of returning to school Entre la escuela obligatoria y la formación post-obligatoria, un enfoque sociocultural de la trayectoria escolar para comprender las dinámicas de retención escolar
}

\author{
Léonie Liechti
}

Volume 47, numéro 1, printemps 2019

Entre décrochage(s) et raccrochage(s) scolaires : paroles de jeunes et mises en récit

URI : https://id.erudit.org/iderudit/1060854ar

DOI : https://doi.org/10.7202/1060854ar

Aller au sommaire du numéro

Éditeur(s)

Association canadienne d'éducation de langue française

ISSN

1916-8659 (numérique)

Découvrir la revue

Citer cet article

Liechti, L. (2019). Entre école obligatoire et formation postobligatoire. Une approche socioculturelle de la trajectoire scolaire pour comprendre les dynamiques d'accrochage scolaire. Éducation et francophonie, 47(1), 183-204. https://doi.org/10.7202/1060854ar

\section{Résumé de l'article}

Cette contribution propose d'examiner les dynamiques d'accrochage scolaire à travers l'étude de cas d'une élève inscrite durant une année dans un dispositif suisse de soutien à la transition entre école obligatoire et formation postobligatoire. Dans une visée compréhensive et en adoptant une approche psychoculturelle du développement, cette contribution s'intéresse à l'évolution du sens que l'élève attribue à son expérience scolaire et à son orientation vers le dispositif, tout en considérant la trajectoire scolaire et l'environnement de l'établissement dans lesquels sa situation s'inscrit. Les données utilisées sont issues de quatre entretiens semi-directifs menés avec l'élève (trois durant l'année scolaire 2014-2015 alors qu'elle a 16 ans et fréquente le dispositif, et un en 2017 alors qu'elle a 18 ans). Une analyse biographique de la trajectoire révèle d'abord un désengagement scolaire de l'élève, entraîné par la rupture que provoque l'attribution, par l'établissement d'enseignement, d'un profil scolaire faible à ses 12 ans, puis un réinvestissement scolaire durant son année dans le dispositif. De plus, l'étude du sens que l'élève élabore autour de son expérience scolaire durant l'année de transition révèle l'importance de l'élaboration de sens dans l'accrochage scolaire de l'élève.

Tous droits réservés @ Association canadienne d'éducation de langue française, Ce document est protégé par la loi sur le droit d’auteur. L’utilisation des 2019 services d'Érudit (y compris la reproduction) est assujettie à sa politique d'utilisation que vous pouvez consulter en ligne.

https://apropos.erudit.org/fr/usagers/politique-dutilisation/ 


\title{
Entre école obligatoire et formation postobligatoire. Une approche socioculturelle de la trajectoire scolaire pour comprendre les dynamiques d'accrochage scolaire
}

\author{
Léonie LIECHTI
}

Université de Neuchâtel, Neufchâtel, Suisse

\section{RÉSUMÉ}

Cette contribution propose d'examiner les dynamiques d'accrochage scolaire à travers l'étude de cas d'une élève inscrite durant une année dans un dispositif suisse de soutien à la transition entre école obligatoire et formation postobligatoire. Dans une visée compréhensive et en adoptant une approche psychoculturelle du développement, cette contribution s'intéresse à l'évolution du sens que l'élève attribue à son expérience scolaire et à son orientation vers le dispositif, tout en considérant la trajectoire scolaire et l'environnement de l'établissement dans lesquels sa situation s'inscrit. Les données utilisées sont issues de quatre entretiens semi-directifs menés avec l'élève (trois durant l'année scolaire 2014-2015 alors qu'elle a 16 ans et fréquente le dispositif, et un en 2017 alors qu'elle a 18 ans). Une analyse biographique de la trajectoire révèle d'abord un désengagement scolaire de l'élève, entraîné par la rupture que provoque l'attribution, par l'établissement d'enseignement, d'un profil scolaire faible à ses 12 ans, puis un réinvestissement scolaire durant son année dans 
Entre école obligatoire et formation postobligatoire.

Une approche socioculturelle de la trajectoire scolaire pour comprendre les dynamiques d'accrochage scolaire

le dispositif. De plus, l'étude du sens que l'élève élabore autour de son expérience scolaire durant l'année de transition révèle l'importance de l'élaboration de sens dans l'accrochage scolaire de l'élève.

\section{ABSTRACT}

\section{Between compulsory schooling and post-compulsory training. A sociocultural approach to the educational trajectory in to understand the dynamics of returning to school}

This contribution examines the dynamics of school attendance through the case study of a student enrolled for one year in a Swiss support system for the transition from compulsory schooling to post-compulsory training. By adopting a comprehensive and psycho-cultural approach to development, this paper focuses on the evolution of the meaning the student gives to the school experience and the orientation towards it, while considering academic trajectory and school environment. The data come from four semi-structured interviews conducted with the student (three during the 2014-2015 school year when she was 16 years old and was attending school and one in 2017 when she was 18 years old). First, a biographical analysis of the trajectory shows academic disengagement resulting in dropping out, triggered by the attribution of a weak school profile at age 12 and followed by an academic reinvestment during her year at the establishment. In addition, a study of the meaning the student attributes to her academic experience over the transition year suggests the importance of creating meaning in the student's process of returning to school.

\section{RESUMEN}

Entre la escuela obligatoria y la formación post-obligatoria, un enfoque sociocultural de la trayectoria escolar para comprender las dinámicas de retención escolar

La presente contribución se propone examinar las dinámicas de retención escolar a través del estudio de caso de una alumna inscrita durante un año en un dispositivo suizo de apoyo a la transición entre la escuela obligatoria y la formación post-obligatoria. De manera comprensiva y adaptando un enfoque sicocultural del desarrollo, la presente contribución se interesa a la evolución del significado que el alumno atribuye a su experiencia escolar y a su orientación hacia un el dispositivo, considerando al mismo tiempo la trayectoria escolar y el entorno del establecimiento en el cual la situación está inscrita. Los datos utilizados provienen de cuatro entrevistas semi-directivas realizadas con la alumna (tres durante el año escolar 2014-2015 cuando tenía 16 años y frecuentaba el dispositivo, y una en 2017 cuando tenía 18 años). Un análisis biográfico de la trayectoria muestra por principio un abandono escolar de la alumna, 
Entre école obligatoire et formation postobligatoire.

Une approche socioculturelle de la trajectoire scolaire pour comprendre les dynamiques d'accrochage scolaire

ocasionado por la ruptura que provoca la atribución, de parte del establecimiento, de un perfil escolar bajo a sus 12 años, seguidamente una reinversión escolar durante su año en el dispositivo. Además, el estudio del significado que la alumna elabora en torno a su experiencia escolar durante el año de transición revela la importancia de la elaboración del significado en la retención escolar de la alumna.

\section{INTRODUCTION}

Dans cette contribution, les processus de décrochage et d'accrochage scolaires sont approchés sous l'angle de la transition entre la fin de la scolarité obligatoire et l'entrée dans une formation postobligatoire initiale (ou dans le monde du travail), et plus spécifiquement par l'intermédiaire des réponses de l'établissement d'enseignement, des dispositifs ou des programmes pédagogiques mis en place pour soutenir l'entrée des élèves dans une formation ultérieure.

Depuis quelques décennies, la lutte contre l'échec scolaire et le décrochage dans les pays occidentaux s'est accompagnée de l'émergence de dispositifs divers visant à favoriser l'accrochage scolaire ou la poursuite des études, et ayant en commun la caractéristique «de constituer une alternative ponctuelle, d'une manière ou d'une autre, au fonctionnement ordinaire de l'école» (Barrère, 2013, p. 100). Parallèlement, dans la recherche sur le décrochage, les travaux se sont progressivement intéressés aux dispositifs d'accrochage en cherchant à identifier et à comprendre les éléments de l'environnement de l'établissement favorables comme défavorables à la mobilisation scolaire et au fonctionnement d'un dispositif d'accrochage (Bernard et Michaut, 2009; Blaya, 2012; Blaya, Tièche Christinat et Angelucci, 2019; Bonnéry et Renard, 2013).

Les processus d'accrochage, dans leur dimension subjective, en tant qu'expériences d'engagement ou de mobilisation dans les apprentissages et la scolarité, restent encore peu explorés. Pourtant, la prise en compte de l'expérience subjective de l'élève s'avère cruciale tant pour l'examen des réponses des établissements d'enseignement à la lutte contre le décrochage que pour comprendre les processus d'accrochage scolaire, compte tenu du rôle du rapport de l'élève à l'école et au savoir, et du sens de l'expérience scolaire dans les apprentissages et dans la poursuite de la scolarité ou d'une formation (Assude, Feuilladieu et Dunand, 2015; Bautier, 2003; Flavier et Méard, 2016; Prêteur, Constans et Féchant, 2004). En outre, les questions relatives à l'expérience subjective des élèves semblent particulièrement saillantes en ce qui concerne spécifiquement leur orientation scolaire à l'issue de la scolarité obligatoire, tout particulièrement lorsque celle-ci peut sembler davantage contrainte ou forcée 
et que les élèves sont appelés à en faire un projet personnel (Léonardis, CapdevielleMougnibas et Prêteur, 2006).

À l'instar de ces travaux qui appréhendent la difficulté scolaire comme émergeant des interactions entre l'élève et son environnement, cette contribution propose d'examiner la trajectoire scolaire d'une élève, Belen, inscrite à 16 ans dans une «année de transition», un dispositif suisse de soutien vers une formation postobligatoire. Pour ce faire, l'étude de cas développée ici est orientée par deux objectifs de recherche, qui sont: 1) d'identifier les éventuelles ruptures sur le plan scolaire et de situer l'inscription de l'année transitoire dans la trajectoire de l'élève; 2) d'explorer comment le sens attribué à son année de transition et à son orientation vers le dispositif évolue à travers le temps et intervient dans les dynamiques d'accrochage scolaire.

\section{DÉCROCHAGE ET TRANSITIONS SCOLAIRES EN SUISSE: QUELQUES SPÉCIFICITÉS}

En Suisse, dans les cantons francophones (ou romands), la scolarité obligatoire dure onze ans et est composée du degré primaire (huit ans) et du degré secondaire I, ou école secondaire (trois ans). À l'issue des huit années de la scolarité primaire (vers 12 ans), au moment d'entamer les trois années du degré secondaire I, l'élève est assigné à une filière plus ou moins exigeante en fonction de son profil scolaire. Celui-ci est établi sur la base de ses compétences scolaires en français, en mathématiques et en allemand, elles-mêmes évaluées par des tests cantonaux standardisés durant la dernière année de la scolarité primaire. À la fin du degré secondaire I, vers 16 ans, l'élève peut s'orienter vers différentes formations du degré secondaire II (degré postobligatoire), dont les écoles préparant aux études (universités et hautes écoles) et à la formation professionnelle, et ce, en école ou en apprentissage (alliant école et formation en entreprise). L'entrée dans une formation postobligatoire initiale est toutefois fortement contrainte par la filière suivie par l'élève au degré secondaire I ainsi que par ses résultats scolaires à l'issue de l'école obligatoire.

Cette sélection précoce des élèves dans leur parcours scolaire est reconnue comme mettant à mal l'équité dans la réussite scolaire en Suisse (Centre suisse de la coordination pour la recherche en éducation [CSRE], 2014; Perini, 2012) et comme traçant les grandes lignes de leur parcours ultérieur (Pagnossin et Armi, 2011). De plus, si près d'un quart des élèves rencontrent des difficultés à accéder directement à une formation postobligatoire à l'issue de leur scolarité (CSRE, 2014), cette précarité dans l'orientation des élèves s'observe également en fin de scolarité obligatoire, en lien avec leur profil scolaire: parmi les quelque $5 \%$ d'élèves qui n'ont pas entamé une formation initiale dans les deux années suivantes, celles et ceux qui ont fréquenté les filières du degré secondaire I les moins exigeantes y sont largement représentés (Office fédéral de la statistique, 2016). 
Dans le paysage éducatif, de nombreuses réponses d'établissements d'enseignement ont émergé depuis plus d'une vingtaine d'années pour soutenir l'entrée des élèves dans une formation initiale. Fortement hétérogènes, ces mesures varient entre elles selon le public visé, leur degré d'institutionnalisation, leur durée, les acteurs impliqués, ou encore selon l'accent mis par le programme tantôt sur la consolidation des acquis scolaires, tantôt sur la préparation à l'entrée dans le monde du travail. Mais il est aussi reproché à ces dispositifs d'être plutôt conçus pour combler les lacunes des élèves que pour les soutenir dans leurs propres parcours, questionnements et choix durant la transition vers une formation initiale (Lambelet, 2014; Masdonati, 2007; Masdonati et Zittoun, 2012).

\section{UNE APPROCHE PSYCHOCULTURELLE DE LA TRAJECTOIRE SCOLAIRE}

Pour examiner les trajectoires scolaires et l'évolution du sens de l'expérience scolaire sans perdre de vue leurs dimensions sociales et culturelles, j'adopte ici une perspective socioculturelle du développement. Admettant l'irréductibilité et l'irréversibilité du temps (Valsiner, 1994), la personne est considérée dans une telle perspective comme un acteur en constante interaction avec l'environnement socioculturel (et donc avec d'autres personnes, réelles ou imaginaires, des objets et des éléments culturels, des symboles, etc.), qui va dessiner une trajectoire unique au cours de sa vie. Cette approche invite à porter une attention particulière aux processus par lesquels une personne comprend le monde et attribue du sens aux expériences vécues, tout en considérant leur nature socialement et culturellement médiatisée (Valsiner, 2014). Porté sur un phénomène éducatif, ce regard s'avère particulièrement pertinent du fait de considérer tant les dimensions intrapsychiques (comme les processus d'apprentissage et de construction de sens des élèves) que les composantes sociales et culturelles d'un environnement scolaire spécifique (Perret-Clermont, 2015; Zittoun, 2016).

\section{Trajectoires : histoires personnelles et cheminements objectifs}

La notion de trajectoire est ici mobilisée pour conceptualiser l'histoire scolaire de l'élève sous un angle psychoculturel et développemental. De ce fait, elle est envisagée comme l'histoire individuelle, multilinéaire et non prédictible du développement d'une personne tout au long de sa vie (Zittoun et Gillespie, 2015b), située historiquement et émergeant en composant avec les normes et les demandes d'un environnement socioculturel qui favorise ou entrave les possibilités d'actions (Elder et Shanahan, 2006). Par conséquent, la trajectoire renvoie non seulement au cheminement objectif d'un individu pouvant être décrit sur la base d'événements socialement reconnus (tels l'entrée à l'école ou un redoublement), mais aussi au parcours subjectif, à l'histoire vécue de la personne. De plus, les trajectoires individuelles ne sont pas stables et, parmi les changements qui s'y opèrent, les moments de rupture, 
qui «mett[ent] en question l'allant de soi de leur expérience quotidienne» (Zittoun, 2008a, p. 47), sont plus susceptibles d'entraîner une réélaboration profonde de la compréhension de la personne et de son rapport à l'environnement.

\section{Élaboration de sens et expérience scolaire}

Le concept de sens en psychologie socioculturelle repose sur la distinction, proposée par Vygotsky (1962), entre signification sociale, partagée, et sens personnel ou subjectif qui s'enracine dans l'histoire individuelle et la subjectivité de la personne. Dans cette idée, tout objet que l'individu s'approprie et autour duquel il développe un sens qui lui est propre est d'abord porteur d'une signification sociale, plutôt stable, ellemême fonction de son ancrage social, culturel et historique.

Plus qu'une simple association ou intégration de la réalité objective par la personne, le processus de construction de sens ou de symbolisation est complexe. Un tel travail d'élaboration symbolique et d'intégration de l'expérience vécue demande en effet de pouvoir «lier le nouveau, l'ici et maintenant, avec du connu, du déjà-là, du pensé avant» (Zittoun, 2013, p. 227). Ainsi, par les processus de construction de sens, la personne interprète, à l'aide de ses connaissances préalables et de son expérience personnelle, les situations, les objets, les discours ou les événements, et développe des représentations plus ou moins réalistes de ceux-ci pour comprendre ce qui lui arrive (Zittoun et Brinkmann, 2012). Néanmoins, la construction de sens suppose que la personne puisse se distancer a minima de l'expérience vécue pour la relier à d'autres situations, processus qui participe à un sentiment de continuité et facilité par la mise en récit (Masdonati et Zittoun, 2012).

Dans le domaine scolaire, l'étude du sens et de ses implications dans les processus d'apprentissage est fréquemment abordée en référence à la notion d'expérience scolaire développée par Rochex (1998). Souvent liée à la notion de rapport au savoir et à l'école, elle propose d'articuler une dimension plutôt objective de la scolarité (les curricula, les objets de savoir, les contenus, etc.) avec une dimension plus subjective liée au sens personnel de la scolarité et des projets de formation que développe un élève, ou aux raisons qui sous-tendent son engagement dans les apprentissages. Plus récemment, le sens de l'expérience scolaire des élèves a été étudié en lien avec leur projet de formation et leur orientation professionnelle, considérant non seulement le sens attribué à leur scolarité préalable (l'autoévaluation de leur scolarité) et à leurs représentations de soi en tant qu'élèves, mais aussi le sens attaché à leur orientation professionnelle et à leur relation à l'avenir (Capdevielle-Mougnibas et CourtinatCamps, 2017; Léonardis et al., 2006).

Dans l'orientation plus sémiotique adoptée ici, l'élaboration de sens est envisagée comme une dynamique dialogique entre la personne et le monde social (Lawrence et Valsiner, 2003; Zittoun et Gillespie, 2015a). En reconnaissant la construction de 
sens comme un processus psychologique d'interprétation du monde socialement et culturellement guidé, j'emprunte ici le modèle du prisme sémiotique développé par Zittoun (2006) pour examiner les processus de construction et de réélaboration du sens personnel autour de l'expérience scolaire. Celui-ci inclut quatre pôles: la personne, l'autre, l'objet et le sens personnel; les côtés du prisme sont ainsi les relations qui unissent ces éléments. Ce modèle a l'avantage de représenter tant les processus interpersonnels (personne - autre) que les processus intrapersonnels ou intrapsychiques (personne - sens personnel). Considéré de manière dynamique, à savoir comme un arrêt sur image de processus en constante évolution, où les autres et les objets avec qui la personne interagit varient, ce prisme peut être vu comme représentant les différentes positions que la personne adopte à travers le temps. D'une position initiale, la personne peut s'engager dans des processus de décentration qui l'amènent à conférer du sens à un objet. Mais elle peut aussi reconfigurer ce sens, impliquant alors une nouvelle prise de distance avec l'objet pour pouvoir le nommer, le signifier, voire le communiquer (Zittoun, 2008b).

\section{CADRE MÉTHODOLOGIQUE}

Létude de cas présentée ici est tirée d'une recherche plus large menée au sein d'un dispositif éducatif de soutien à la transition vers la formation postobligatoire dans un canton de Suisse romande. Ce dispositif est intégré à un établissement de formation du degré secondaire II et s'inscrit dans l'offre cantonale des formations postobligatoires. Il est destiné aux élèves de 16 ans et plus qui ont terminé leur scolarité obligatoire sans pouvoir entrer dans une formation postobligatoire initiale et accueille ainsi durant une année une cinquantaine d'élèves souhaitant mûrir leur choix d'orientation et consolider leurs acquis scolaires. Le programme proposé est ainsi axé prioritairement sur le renforcement des compétences scolaires en vue de permettre aux élèves d'intégrer une formation postobligatoire l'année suivante, et les principaux intervenants sont membres de l'équipe enseignante. Le programme comprend principalement l'enseignement des disciplines scolaires usuelles (français, mathématiques, etc.), mais aussi des ateliers, des cours plus manuels, appliqués ou artistiques (théâtre, informatique, chimie, etc.) ainsi que des moments dévolus au développement et au suivi de l'orientation des élèves (ateliers de soutien dans les différentes démarches, stages, permanence de psychologues de l'orientation, etc.).

Dans le but d'explorer cet environnement éducatif spécifique dans ses dimensions socioculturelles et subjectives, l'ensemble du corpus de données a été constitué en variant les sources et en utilisant différentes méthodes, permettant ainsi une triangulation des données et offrant une base au processus de généralisation à partir d'études de cas (Zittoun, 2017): observation en classe, discussions informelles et entretiens d'experts avec divers acteurs impliqués, récolte de traces et documents de l'établissement, et entretiens semi-directifs avec des membres du corps enseignant ainsi qu'avec des élèves du dispositif. 
Les données utilisées ici ont été recueillies dans le cadre du volet de la recherche qui s'intéresse à la perspective des élèves, par le biais d'entretiens semi-directifs. Le corpus considéré pour cette étude de cas est constitué des données issues de quatre entretiens de recherche menés avec Belen. Les trois premiers se sont déroulés dans les locaux de l'établissement de formation qui accueille le dispositif étudié alors que la jeune fille a 16 ans, au terme de chaque trimestre de l'année scolaire 2014-2015, soit en décembre (T1), avril (T2) et juin (T3). Le dernier entretien de recherche (T4) s'est tenu au domicile de l'élève en février 2017 (18 mois après l'année de transition) alors qu'elle a 18 ans.

Les grilles de chaque entretien (synthèse en annexe) ont été construites sur la base des approches narratives et biographiques de conduite d'entretiens, et elles ont été particulièrement orientées par les travaux contemporains qui placent au centre de leur démarche l'expérience et la subjectivité de la personne (Hviid, 2008, 2012). Le premier entretien consistait principalement à inviter l'élève à raconter son parcours scolaire et son arrivée dans le dispositif. Le second portait davantage sur le vécu et les expériences de l'élève durant son année dans le dispositif. Le troisième entretien, en fin d'année scolaire, proposait principalement à l'élève d'établir un bilan de l'année écoulée. Le dernier entretien visait, d'une part, à compléter le parcours de l'élève depuis sa sortie du dispositif et, d'autre part, à recueillir les propos de l'élève sur son expérience préalable dans le dispositif. Les quatre entretiens avec Belen, enregistrés et retranscrits, ont duré entre 20 (T3, bref bilan de fin d'année) et 150 minutes (T4).

La démarche d'analyse des données, entreprise en deux temps, s'inspire de l'approche biographique des trajectoires (Rosenthal, 1993). Le but de la première étape est de reconstruire la trajectoire scolaire de l'élève en reconstituant la suite chronologique d'événements et d'expériences scolaires de son récit. J'ai été ici attentive aux remarques explicites sur le temps, aux indicateurs de changement, aux marqueurs temporels et aux éléments culturels qui leur sont liés dans le récit de l'élève.

Pour la seconde étape, une analyse thématique a d'abord permis d'identifier, puis de retenir les segments pour l'analyse de l'élaboration de sens. Dans une démarche interprétative et à l'aide de l'analyse préalable de la trajectoire, une attention particulière a ensuite été portée aux segments présentant des indices d'une mise à distance, dans le récit de l'élève, de l'expérience scolaire et de l'orientation rattachées au dispositif, notamment l'usage de métaphores, la mise en lien d'expériences passées, présentes et futures, la réflexion sur sa situation et son orientation, ou encore la mise en lien de son expérience scolaire avec d'autres personnes (Zittoun, 2014). 


\section{ÉTUDE DE CAS}

Dans un premier temps, je présente succinctement la trajectoire scolaire de Belen et ses principaux événements scolaires. Les observations principales issues de l'analyse interprétative sont ensuite exposées pour rendre compte de l'évolution du sens que l'élève attribue au fil du temps à son expérience dans le dispositif de transition.

\section{Trajectoire scolaire}

Née en Suisse de parents immigrés, Belen évoque une scolarité difficile dans son ensemble, mais se définit comme une bonne élève, ce qu'elle illustre par l'absence de sanctions scolaires ou encore de relations conflictuelles avec ses pairs ou le corps enseignant durant son parcours. À la suite des épreuves cantonales de fin de scolarité primaire, à 12 ans, le profil scolaire le moins exigeant (3-3-3 $\left.{ }^{1}\right)$ lui est assigné pour entrer au degré secondaire I.

À l'issue du degré secondaire I, Belen n'a effectué aucun stage, ne trouve pas de place d'apprentissage et ne peut prétendre à aucune formation postobligatoire en raison de son profil et de ses résultats scolaires. Elle souhaite alors travailler dans un bureau comme employée de commerce et entretient depuis son enfance le rêve de devenir avocate. À la fin de sa scolarité obligatoire, elle s'inscrit à l'année de transition sur les recommandations conjointes des services cantonaux d'orientation scolaire et professionnelle, impliqués dans la procédure d'inscription, et de sa sœur. La perspective d'une année dans un dispositif de transition ne la réjouit pas et, durant le premier mois dans le dispositif, découragée, elle remet en question sa présence dans celui-ci. Elle reconnaît progressivement ses propres difficultés scolaires et choisit de s'investir durant son année dans le dispositif. Lors du premier entretien de recherche, elle présente le dispositif de transition comme «l'occasion de recommencer à zéro, [...] de tout mettre de côté et de refaire une année blanche, [...] comme si j'avais rien fait durant au moins quinze ans et cette année [...] c'est une nouvelle année.» (T1)

Belen effectue ensuite trois stages en entreprise durant l'année, et ses résultats scolaires s'améliorent, lui ouvrant l'accès à deux écoles du degré secondaire II. Avant le dernier trimestre de l'année scolaire, elle obtient une place d'apprentissage dans une entreprise et s'oriente vers une formation professionnelle duale, laquelle correspond davantage, selon elle, à ses compétences scolaires, à son intérêt pour l'école et à son projet initial de formation.

1. Attribué sur la base des résultats scolaires durant la dernière année d'école primaire dans les trois disciplines fondamentales (français, mathématiques et allemand), le profil scolaire indique le degré de compétence de l'élève de 1 à 3 (" 3 » représentant le degré de compétence le moins exigeant) dans chacune des trois disciplines. 
Elle commence son apprentissage en août 2015, mais se heurte rapidement à ce qu'elle qualifie d' "échec scolaire»: après quatre semaines de formation, elle est convoquée par la direction de l'établissement, car elle ne satisfait pas aux exigences scolaires de sa filière. Avec l'appui de sa famille et de son entreprise formatrice, elle s'investit, fournit beaucoup d'efforts, se remet profondément en question quant à son orientation et à son choix de formation postobligatoire, et parvient «miraculeusement», selon ses termes, à améliorer ses résultats scolaires. Lors du dernier entretien de recherche, un peu plus d'une année après avoir amorcé sa formation initiale, Belen se dit fatiguée, stressée, mais très motivée à réussir sa formation.

\section{Rupture scolaire et orientation vers le dispositif de transition}

Dans la trajectoire scolaire de Belen, une rupture nette apparaît, concrétisée par l'attribution du profil scolaire le moins exigeant à l'issue de sa dernière année d'école primaire. Cette dernière, «la pire» année selon ses propos, représente pour elle une injustice lourde de conséquences sur son orientation scolaire et professionnelle. Ainsi, les trois années au degré secondaire I sont marquées pour l'élève par un désengagement scolaire.

"Ça ruine pas notre vie, mais ça nous fait un choc d'être [3-3-3], c'est difficile quoi [...] mais c'est pour ça les jeunes, j'pense la plupart en tout cas, moi j'travaillais pas à cause de ça, j'étais [3] de toute façon. Est-ce que j'peux être moins? Oui, j'peux être en [classe d'enseignement spécialisé], mais non, j'pouvais pas.» (T1)

Cet extrait illustre, dans le récit de Belen, un aspect central de cet épisode critique et de la démobilisation scolaire qui s'ensuit, et renvoie au processus d'étiquetage de l'élève par les établissements de formation. Ce désengagement, qui se précise dès son entrée dans l'enseignement secondaire I, se reflète également dans la façon dont Belen développe son projet de formation. Dès la moitié de son parcours dans le degré secondaire I, la seule orientation qu'elle envisage est une formation postobligatoire à laquelle elle ne peut pourtant prétendre en raison de ses résultats scolaires. De plus, elle commence à postuler pour des places d'apprentissage en entreprise peu avant la fin de sa scolarité obligatoire, alors qu'elle n'a fait aucun stage. Finalement, pour Belen, l'orientation vers le dispositif de transition représente non seulement une des conséquences de son étiquetage scolaire, mais aussi la seule alternative envisageable pour éviter un redoublement ou une prolongation de la scolarité. 
Entre école obligatoire et formation postobligatoire.

\section{Sens en mouvement: de l'échec à la réussite}

Dans un premier temps, Belen perçoit négativement son orientation vers le dispositif de transition à l'issue de sa scolarité obligatoire (un «échec», une "année déchet», un «redoublement» «pour ceux qui [n']avaient pas leur place»). Ce sens personnel du dispositif de transition rejoint une signification plus largement partagée et relayée, selon Belen, par certains parents, la plupart des élèves et des membres du corps enseignant des degrés I et II du secondaire, ou encore de potentiels employeurs.

Au cours de l'année transitoire s'opère un changement dans le sens qu'elle attribue à son inscription dans le dispositif. Celui-ci représente peu à peu pour elle une «dernière chance» à ne pas «gâcher», une occasion qui lui est offerte de «s'améliorer». Ce glissement du sens personnel qu'elle attribue à son inscription est favorisé, dans son récit, par une double dynamique. D'abord, cette transformation du sens personnel est socialement orientée vers d'autres élèves: ceux, réels, inscrits avec elle dans le dispositif, et ceux, imaginés, qui n’ont pas été admis dans celui-ci.

«Après qu'on a vraiment été dans nos classes avec les autres durant une année, [...] j'ai vu dans les yeux de tout l'monde un peu le même sentiment que moi, que ben ils avaient pas de place d'apprentissage, qu'y devaient beaucoup travailler à ce niveau, donc c'est pour ça que j'me suis dit "ben accroche-toi parce que t'as une chance, y prennent déjà pas tout le monde parce qu'y a beaucoup de postulations pour [le dispositif de transition], donc ils t'ont pris, ça veut dire que voilà, $\mathrm{y}$ te donnent une dernière chance pour réussir, donc heu pourquoi pas la saisir quoi", donc c'est pour ça j'ai vraiment voulu m'accrocher, c'est ce déclic.» (T3)

La mise à distance et la réévaluation de l'expérience de Belen s'effectuent ici en référence à différentes figures d'élèves, apportant des repères pour repenser sa propre situation scolaire. Selon elle, cette mise en regard entraîne un "déclic» qui l'amène dès lors à «[s]'accrocher» et favorise son engagement dans l'année de transition.

Mais cette évolution du sens personnel de l'expérience scolaire est aussi fortement liée au caractère non obligatoire de sa situation scolaire dans le dispositif, renvoyant ainsi à une caractéristique structurelle: l'inscription de celui-ci dans l'enseignement postobligatoire. De manière intéressante, cette «réalité» fait largement écho aux discours, aux pratiques et aux aménagements du corps enseignant, et fait plus généralement référence aux différents choix opérés par l'élève durant l'année de transition:

«Déjà on n'est pas obligé. Ça, on nous le dit clairement et heu quand y voient que on veut pas être là, y nous dit clairement qu'on n'est pas obligé d'être ici que voilà, c'est notre choix. Ça, c'est déjà quelque chose d'énorme parce qu'on est face à une réalité quand même, si on est ici, oui peut-être 
on est obligés par nos parents, mais on est vraiment obligé parce qu'on l'a voulu.» (T3)

Dans la nouvelle lecture de sa situation illustrée dans cet extrait, l'élève fait référence à son propre "choix", qui semble inhérent, dans son discours, à son inscription dans le dispositif. De plus, le rôle que semble ici jouer le corps enseignant («on nous le dit», «y voient») dans la considération de cette caractéristique coïncide avec les récits d'autres élèves, les observations faites en classe et les témoignages de certains des membres du corps enseignant.

Dans sa dimension plus subjective, l'inscription non obligatoire dans le dispositif apparaît comme un élément central de son expérience scolaire durant l'année dans le dispositif, un préalable à son réinvestissement scolaire. D’abord, cette relecture semble favoriser une appréhension plus positive de sa situation du fait de l'inscrire, en tant qu'élève de première année de formation postobligatoire, et non pas dans une situation assimilable à un redoublement.

Ensuite, la caractéristique non obligatoire liée à la situation de l'élève apparaît comme un moteur important pour réfléchir à son orientation, considérer ses champs d'intérêt, ses possibilités et ses responsabilités, tout en envisageant les contraintes et les défis à relever pour concrétiser ses souhaits de formation. Rétroactivement, son expérience dans le dispositif représente plus clairement pour Belen une "première réussite» (T4) dans sa trajectoire, tant sur le plan des apprentissages scolaires que sur celui de son orientation et de son projet de formation, le caractère non obligatoire restant un élément saillant dans son récit. Ainsi, le dispositif de transition représente pour l'élève une occasion ou une dernière chance, qu'elle a saisie tant pour évoluer sur le plan scolaire (apprendre à travailler de manière autonome, pour elle-même, et à persévérer dans les apprentissages) que pour effectuer un choix plus réfléchi «qui dirigera [sa] vie» (T4) (l'orientation vers le degré secondaire II).

L'année de transition représente ainsi un épisode charnière de sa trajectoire scolaire, car elle vient contrecarrer, par l'ouverture de diverses voies de formation notamment, une orientation perçue comme subie.

« [N]ous à l'école obligatoire ou même encore à l'école, maintenant, ils nous donnent les ingrédients, ils nous apprennent à le faire et, derrière nous, ils le contrôlent et quand c'est faux en plus de ça, ils le cassent, ils le jettent, et on doit recommencer à nouveau [...], c'est à dire que quand t'es mauvais à l'école, ils te redoublent. C'est eux qui choisissent quoi faire de ta vie, alors que ça, j'trouve c'est dégueulasse parce que c'est toi qui dois choisir c'que tu veux faire pour pas par la suite regretter, alors qu'à [l'année de transition], c'était pas comme ça. On te donnait les ingrédients [...], on te montrait pas comment il fallait faire, on te donnait de l'aide pour le faire, 
mais on te montrait jamais ce qu'il fallait faire pour que tu puisses faire toi ton propre gâteau, à ton goût ou pas.» (T4)

La métaphore que propose ici Belen alors qu'elle est apprentie illustre le sens de son expérience scolaire dans le dispositif, qui se dessine dans un fort contraste avec ses expériences plus ordinaires, passées et actuelles.

\section{DISCUSSION ET REMARQUES CONCLUSIVES}

En m'intéressant à la trajectoire d'une élève inscrite dans un dispositif de soutien à la transition et au sens qu'elle élabore autour de son expérience scolaire, j'ai souhaité illustrer le potentiel qu'une approche socioculturelle et biographique peut offrir pour examiner les dynamiques de décrochage et, surtout, d'accrochage scolaires. La principale limite de l'étude réside d'ailleurs dans la présentation d'une trajectoire dans laquelle l'accrochage scolaire est favorisé par la fréquentation d'un dispositif. Si elle est emblématique des autres élèves de la recherche pour qui l'année de transition représente une occasion, elle laisse dans l'ombre les cas d'élèves pour qui le même dispositif n'implique pas une telle mobilisation.

Toutefois, l'analyse a d'abord permis de mettre en évidence une rupture (l'attribution d'un profil scolaire) dans la trajectoire de l'élève, couplée à un sentiment d'injustice à l'égard de sa scolarité. Venant corroborer ces observations, une rupture à un moment de la scolarité ainsi qu'un sentiment d'injustice à l'égard de l'établissement scolaire sont des caractéristiques fréquemment relevées dans les parcours d'élèves en décrochage (Bernard, 2011; Bernard et Michaut, 2014; Pelletier et Alaoui, 2016). De plus, l'origine de cette rupture précédant la démobilisation de l'élève est ici à chercher dans l'organisation et les processus de sélection de l'établissement scolaire, faisant écho aux travaux qui soulignent le poids de certains facteurs scolaires dans le processus de décrochage, dont les pratiques d'étiquetage et de disqualification des élèves employées par les établissements d'enseignement (Blaya, Gilles, Plunus et Tièche Christinat, 2011).

L'analyse du sens de l'expérience scolaire de l'élève a ensuite révélé une évolution, allant d'une évaluation négative de son orientation vers l'année transitoire à une représentation plus positive de celle-ci (une occasion), et symbolisant finalement une première réussite scolaire. Cette transformation dans sa façon de se représenter sa situation ainsi que le réinvestissement scolaire dont elle témoigne durant l'année de transition suggèrent que le travail d'élaboration de sens autour de son expérience scolaire représente une composante primordiale de sa mobilisation scolaire.

Rejoignant ainsi les travaux qui soulignent le rôle majeur attribué au sens de l'orientation et de l'expérience scolaire tant dans la réussite/l'échec scolaire (Bautier et Goigoux, 2004; Courtinat-Camps et Prêteur, 2012) que dans la rupture/poursuite 
d'une formation postobligatoire (Capdevielle-Mougnibas et Courtinat-Camps, 2017), la démarche proposée a cependant permis de déterminer certaines figures sociales, charriant discours et significations susceptibles d'intervenir dans l'élaboration d'un sens personnel. En outre, du fait de reconnaître la dimension temporelle inhérente à tout phénomène développemental, un des apports majeurs de l'approche socioculturelle adoptée ici réside dans le fait de conceptualiser l'élaboration de sens comme un processus socialement et culturellement orienté et dynamique, plutôt qu'un état ou une représentation statique et stable dans le temps.

Ainsi, la transformation du sens personnel est socialement guidée ici par d'autres élèves et le corps enseignant du dispositif. De plus, le caractère non obligatoire de sa situation dans le dispositif (inhérent à l'ancrage institutionnel de celui-ci dans la formation postobligatoire) en représente une composante saillante. Faisant écho aux mises en garde émises à l'endroit des établissements, des mesures et des pratiques éducatives actuelles à travers lesquelles sont susceptibles d'opérer les nouveaux mécanismes de domination tels que l'injonction à l'autonomie, à l'indépendance ou à la participation (Martuccelli, 2004), cette observation mérite d'être abordée avec prudence. En effet, les questions de choix et d'obligation, ici véhiculées par le corps enseignant et intervenant dans l'élaboration d'un sens personnel de l'expérience, attirent l'attention sur les enjeux éthiques contemporains qui marquent ainsi tout particulièrement les réponses mises en place par les établissements d'enseignement pour soutenir les élèves dans leur transition à l'issue de la scolarité.

Si cet élément s'avère crucial dans la réflexion sur les pratiques et les mesures pédagogiques en place tout en s'accompagnant de nombreux paradoxes, il implique de pouvoir mieux déterminer des pistes d'action qui favorisent davantage l'émancipation de l'élève que sa subordination. De ce fait, il est intéressant de relever finalement que cette inscription non obligatoire de la situation de Belen favorise bien chez elle une réflexion sur ses responsabilités, ses choix et son autonomie quant à sa scolarité et à son parcours. Rétrospectivement, ce caractère «choisi» marque d'autant plus fortement l'expérience de l'élève dans le dispositif que celle-ci permet une orientation non forcée vers une formation du degré secondaire II, et semble ainsi plutôt favoriser une certaine résistance à l'orientation contrainte par les expériences scolaires préalables.

\section{Références bibliographiques}

ASSUDE, T., FEUILLADIEU, S. et DUNAND, C. (2015). Conditions d'évolution du rapport au savoir mathématique de jeunes «décrocheurs». Carrefours de l'éducation, 40(2), 167-182. https://doi.org/10.3917/cdle.040.0167 
BARRÈRE, A. (2013). La montée des dispositifs: un nouvel âge de l'organisation scolaire. Carrefours de l'éducation, 36(2), 95-116. https://doi.org/10.3917/cdle. 036.0095

BAUTIER, E. (2003). Décrochage, déscolarisation. La nouvelle revue de l'AIS, (24), 33-54.

BAUTIER, E. et GOIGOUX, R. (2004). Difficultés d'apprentissage, processus de secondarisation et pratiques enseignantes: une hypothèse relationnelle. Revue française de pédagogie, 148(1), 89-100. https://doi.org/10.3406/rfp.2004.3252

BERNARD, P.-Y. (2011). Le décrochage des élèves du second degré: diversité des parcours, pluralité des expériences scolaires. Les Sciences de l'éducation - Pour l'Ère nouvelle, 44(4), 75-97. https://doi.org/10.3917/Isdle.444.0075

BERNARD, P.-Y. et MICHAUT, C. (2009). La place de la certification dansle traitement du décrochage scolaire. L'exemple de la Mission générale d'insertion de l'Éducation nationale. Éducation et sociétés, 24(2), 127-142. https://doi.org/10.3917/ es.024.0127

BERNARD, P.-Y. et MICHAUT, C. (2014). «Marre de l'école»: les motifs de décrochage scolaire. Notes du CREN, (17), 1-17.

BLAYA, C. (2012). Le décrochage scolaire dans les pays de l'OCDE. Regards croisés sur l'économie, 12(2), 69-80. https://doi.org/10.3917/rce.012.0069

BLAYA, C., GILLES, J.-L., PLUNUS, G. et TIÈCHE CHRISTINAT, C. (2011). Accrochage scolaire et alliances éducatives: vers une intégration des approches scolaires et communautaires. Education et francophonie, 39(2), 227-249.

BLAYA, C., TIÈCHE CHRISTINAT, C. et ANGELUCCI, V. (dir.). (2019). Au coeur des dispositifs d'accrochage scolaire. Continuité et alliances éducatives. Bruxelles, Belgique: EME.

BONNÉRY, S. et RENARD, F. (2013). Des pratiques culturelles contre l'échec et le décrochage scolaires. Sociologie d'un détour. Lien social et politiques, (70), 135-150. https://doi.org/10.7202/1021160ar

CAPDEVIELLE-MOUGNIBAS, V. et COURTINAT-CAMPS, A. (2017). Forms of school experience in France's vocational training track students. European Journal of Psychology of Education, 32(1), 21-38. https://doi.org/10.1007/s10212-015-0283-4 
Centre suisse de la coordination pour la recherche en éducation (CSRE). (2014). L'éducation en Suisse. Rapport 2014. Aarau, Suisse: CSRE. Repéré à http://www. skbf-csre.ch/fileadmin/files/pdf/bildungsmonitoring/bildungsbericht2014/rapport_ education_2014.pdf

COURTINAT-CAMPS, A. et PRÊTEUR, Y. (2012). Diversité des expériences scolaires chez des collégien(ne)s scolarisés en $3^{\mathrm{e}}$. L'orientation scolaire et professionnelle, 41(4). https://doi.org/10.4000/osp.3918

ELDER, G. H. et SHANAHAN, M. J. (2006). The life course and human development. Dans W. Damon et R. M. Lerner (dir.), Handbook of child psychology. Volume 1. Theoretical models of human development (p. 665-715). Hoboken, NJ : John Wiley \& Sons.

FLAVIER, É. et MÉARD, J. (2016). L'approche du décrochage scolaire selon les théories culturalistes de l'activité. Bénéfices et perspectives. Activités, 13(2). https://doi. org/10.4000/activites.2781

HVIID, P. (2008). Interviewing using a cultural-historical approach. Dans M. Hedegaard et M. Fleer (dir.), Studying children: A cultural-historical approach (p. 139-156). Maidenhead, Royaume-Uni: Open University Press.

HVIID, P. (2012). «Remaining the same» and children's experience of development. Dans M. Hedegaard, K. Aronsson, C. Højholt et O. Skjær Ulvik (dir.), Children, childhood and everydaylife. Children's perspectives (p. 37-52). Charlotte, NC: Information Age Publishing.

LAMBELET, D. (2014). Jeunes en difficulté dans la transition école-métier: ce que le case management veut dire et peut faire. Empan, 93(1), 141-149.

LAWRENCE, J. A. et VALSINER, J. (2003). Making personal sense: An account of basic internalization and externalization processes. Theory \& Psychology, 13(6), 723-752. https://doi.org/10.1177/0959354303136001

LÉONARDIS, M. de, CAPDEVIELLE-MOUGNIBAS, V. et PRÊTEUR, Y. (2006). Sens de l'orientation vers l'apprentissage chez les apprentis de niveau V: entre expérience scolaire et rapport à l'avenir. L'orientation scolaire et professionnelle, 35(1), 5-27. https://doi.org/10.4000/osp.867

MARTUCCELLI, D. (2004). Figures de la domination. Revue française de sociologie, 45(3), 469-497.

MASDONATI, J. (2007). La transition entre l'école et le monde du travail. Préprer les jeunes à l'entrée en formation professionnelle. Bern, Suisse: Peter Lang. 
MASDONATI, J. et ZITTOUN, T. (2012). Les transitions professionnelles. Processus psychosociaux et implications pour le conseil en orientation. L'orientation scolaire et professionnelle, 41(2). https://doi.org/10.4000/osp.3776

Office fédéral de la statistique (OFS). (2016). Analyses longitudinales dans le domaine de la formation. La transition à la fin de l'école obligatoire. Neuchâtel, Suisse: OFS.

PAGNOSSIN, E. et ARMI, F. (2011). Transition entre la formation et le monde du travail: état de la recherche en Suisse. Papers: revista de sociología, 96(4), 1163-1179.

PELLETIER, L. et ALAOUI, D. (2016). Du décrochage provisoire au raccrochage scolaire: l'importance de la reconnaissance. Questions vives. Recherches en éducation, (25), 135-160. https://doi.org/10.4000/questionsvives.1918

PERINI, L. (2012). Peer effects and school design: An analysis of efficiency and equity. Neuchâtel, Suisse: Université de Neuchâtel, Institut de recherche économique. Repéré à ftp://ftp.unine.ch/RePEc/irn/pdfs/wp12-01.pdf

PERRET-CLERMONT, A.-N. (2015). The architecture of social relationships and thinking spaces for growth. Dans C. Psaltis, A. Gillespie et A.-N. Perret-Clermont (dir.), Social relations in human and societal development (p. 51-70). Basingstokes, Royaume-Uni: Palgrave Macmillan.

PRÊTEUR, Y., CONSTANS, S. et FÉCHANT, H. (2004). Rapport au savoir et (dé) mobilisation scolaire chez des collégiens de troisième. Pratiques psychologiques, 10(2), 119-132. https://doi.org/10.1016/j.prps.2004.04.001

ROCHEX, J.-Y. (1998). Le sens de l'expérience scolaire (2e éd.). Paris, France: Presses universitaires de France.

ROSENTHAL, G. (1993). Reconstruction of life stories: Principles of selection in generating stories for narrative biographical interviews. Dans R. Josselson et A. Lieblich (dir.), The narrative study of lives (p. 59-91). Newbury Park, CA: Sage Publications.

VALSINER, J. (1994). Irreversibility of time and the construction of historical developmental psychology. Mind, Culture, and Activity, 1(1-2), 25-42. https:// doi.org/10.1080/10749039409524655

VALSINER, J. (2014). An invitation to cultural psychology. Thousand Oaks, CA: Sage Publications.

VYGOTSKY, L. S. (1962). Thought and language. Cambridge, MA: The MIT Press. 
ZITTOUN, T. (2006). Transitions. Development through symbolic resources. Greenwich, CT: InfoAge.

ZITTOUN, T. (2008a). La musique pour changer la vie. Usages de connaissances, dynamiques de reconnaissance. Éducation et sociétés, 22(2), 43-55.

ZITTOUN, T. (2008b). Learning through transitions: The role of institutions. European Journal of Psychology of Education, 23(2), 165-181.

ZITTOUN, T. (2013). Dans l'intervalle: médiations symboliques et construction du temps. Psychothérapies, 33(4), 225-234.

ZITTOUN, T. (2014). Three dimensions of dialogical movement. New Ideas in Psychology, 32, 99-106. https://doi.org/10.1016/j.newideapsych.2013.05.006

ZITTOUN, T. (2016). Living creatively, in and through institutions. Europe's Journal of Psychology, 12(1), 1-11. https://doi.org/10.5964/ejop.v12i1.1133

ZITTOUN, T. (2017). Modalities of generalization through single case studies. Integrative Psychological and Behavioral Science, 51(2), 171-194. https://doi.org/ 10.1007/s12124-016-9367-1

ZITTOUN, T. et BRINKMANN, S. (2012). Learning as meaning making. Dans N. M. Seel (dir.), Encyclopedia of the sciences of learning (p. 1809-1811). Repéré à http://doc.rero.ch/record/28329?ln=fr

ZITTOUN, T. et GILLESPIE, A. (2015a). Internalization: How culture becomes mind. Culture \& Psychology, 21(4), 477-491.

ZITTOUN, T. et GILLESPIE, A. (2015b). Transitions in the life course. Learning from Alfred Schütz. Dans A. Joerchel et G. Benetka (dir.), Biographical ruptures and their repair: Cultural transitions in development (p. 147-157). Charlotte, NC: Information Age Publishing. 


\section{ANNEXE}

\section{PRÉSENTATION SCHÉMATIQUE DES GRILLES DES QUATRE ENTRETIENS SEMI-DIRECTIFS (ÉLÈVES)}

\section{Introduction (entretiens 1, 2, 3 et 4)}

1. Présentation de l'intervieweuse et du cadre de la recherche

2. Présentation du déroulement de l'entretien, des thèmes abordés et des objectifs spécifiques de l'entrevue

\section{Entretien 1}

1. Présentation de l'élève: sphères d'expérience et activités actuelles Question générale: Comment ta vie actuelle est-elle organisée au quotidien par diverses activités et quelles sont les différentes situations que tu rencontres?

\section{Relances et questions spécifiques}

- Peux-tu expliquer ce qui te plaît dans ces activités et ce que tu apprécies?

- Y a-t-il des loisirs ou des domaines d'activité qui sont plus importants que d'autres pour toi, et pourquoi?

- Quelle place l'école occupe-t-elle dans ta vie aujourd'hui et par rapport à tes différents centres d'intérêt?

$-\ldots$

2. Trajectoire

Question générale: Peux-tu me raconter ton histoire, de ta naissance à ce jour (en pensant par exemple aux événements importants, aux changements, à des épisodes particuliers de ta vie), pour m'expliquer comment tu es devenue la personne que tu es aujourd'hui?

\section{Relances et questions spécifiques}

- Comment s'est déroulée ta scolarité?

- Quels souvenirs as-tu de tes années d'école primaire?

- Peux-tu me raconter comment se sont passées pour toi la fin de l'école primaire et l'entrée à l'école secondaire?

- Quels souvenirs as-tu de tes années au secondaire?

- Quelles sont les différentes périodes ou étapes que tu retraces dans ton parcours (périodes mouvementées, routinières, de changements, etc.)? 
3. Projet de formation et orientation de l'élève

Question générale: Comment se sont développées tes aspirations professionnelles (profession rêvée, métiers envisagés, etc.) jusqu'à aujourd'hui?

\section{Relances et questions spécifiques}

- Quels sont tes projets aujourd'hui en ce qui a trait à ta formation et à ta profession futures?

- Comment t'y prends-tu pour faire avancer tes projets de formation?

- Vers qui ou quoi te tournes-tu lorsque tu as besoin d'aide ou lorsque tu as des doutes, des questions par rapport à ton orientation ou à ton projet?

- Comment t'imagines-tu dans 10 ans?

$-\ldots$

\section{Entretien 2}

1. L'année dans le dispositif

Question générale: Comment se déroule pour toi cette année dans le dispositif depuis ton arrivée ici?

\section{Relances et questions spécifiques}

- Comment voyais-tu ton orientation vers le dispositif au moment de ton inscription en fin d'école obligatoire et quelles étaient tes impressions?

- Quelles étaient tes premières impressions en arrivant dans le dispositif et est-ce qu'elles ont changé?

- Jusqu'à aujourd'hui, qu'est-ce qui te plaît et qu'est-ce qui te plaît moins dans cette année?

- Qu'est-ce que cette année t'a apporté jusqu'à maintenant, qu'est-ce que tu as appris ou pu développer ici?

- Comment sont les relations que tu entretiens avec les enseignantes et les enseignants du dispositif?

$-\ldots$

2. L'élève et l'école

Question générale: Comment te perçois-tu en tant qu'élève (ou étudiante) par rapport à la place de l'école dans ta vie?

Relances et questions spécifiques

- Est-ce que l'école est ou a été importante dans ta vie?

- Quels sont les savoirs et les apprentissages les plus importants que l'école devrait développer chez les élèves, et pourquoi?

- Quels sont les savoirs et les apprentissages les plus importants que tu as pu développer dans ta scolarité? 
- Qu'est-ce que tu aimes ou as aimé apprendre, découvrir, comprendre à l'école ou ici?

$-\ldots$

3. Projet de formation et orientation de l'élève

Question générale: Comment se sont développées tes aspirations professionnelles (profession rêvée, métiers envisagés, etc.) depuis le dernier entretien?

\section{Relances et questions spécifiques}

- Quels sont tes projets aujourd'hui en ce qui a trait à ta formation et à ta profession futures?

$-\ldots$

\section{Entretien 3}

1. L'année dans le dispositif: bilan de l'élève

Question générale: En arrivant à la fin de l'année scolaire, quel bilan tires-tu de cette année?

\section{Relances et questions spécifiques}

- Qu'est-ce que cette année t'a apporté?

- Qu'est-ce que tu attendais de cette année qu'elle n'a pas pu t'offrir?

- Qu'est-ce que ce qui pourrait être modifié selon toi dans le dispositif pour l'améliorer?

- Dans quelle mesure recommanderais-tu à quelqu'un de s'inscrire dans le dispositif?

$-\ldots$

2. Projet de formation et orientation de l'élève

Question générale: Comment se sont développées tes aspirations professionnelles (profession rêvée, métiers envisagés, etc.) depuis le dernier entretien?

\section{Relances et questions spécifiques}

- Quels sont tes projets aujourd'hui en ce qui a trait à ta formation et à ta profession futures?

$-\ldots$ 


\section{Entretien 4}

1. Trajectoire

Question générale: Peux-tu me raconter ton histoire, depuis l'année de transition jusqu'à aujourd'hui (en pensant par exemple aux événements importants, aux changements, à des épisodes particuliers de ta vie)?

\section{Relances et questions spécifiques}

- Comment s'est déroulée ton entrée en formation et quelles étaient tes premières impressions?

- Quels sont les changements les plus importants liés au début de ta formation?

- Est-ce que tu as rencontré des obstacles, des difficultés ou des besoins depuis ton entrée en formation et, dans ce cas, qu'est-ce qui t’a aidée à les surpasser?

$-\ldots$

2. L’année dans le dispositif

Question générale: Que représente ton année dans le dispositif dans ton propre parcours, et quels sont les impressions et les sentiments que tu en gardes aujourd'hui?

\section{Relances et questions spécifiques}

- Dans quelle mesure cette année a-t-elle été importante dans ton parcours de vie?

- Qu'est-ce que cette année t'a apporté de manière générale? En quoi a-t-elle été «utile» ou bénéfique pour la suite de ton parcours?

- Quels sont, selon toi, les avantages et les désavantages liés au fait d'avoir fréquenté le dispositif durant une année?

- Y a-t-il des «choses» (p. ex., savoirs, savoir-faire, compétences, connaissances, etc., liés à l'école ou au projet professionnel, ou des choses plus personnelles) que tu as apprises ou développées durant ton année dans le dispositif et qui te sont toujours utiles aujourd'hui?

- Quels conseils donnerais-tu à un ou une élève qui va commencer son année dans le dispositif?

$-\ldots$

3. Projet de formation et orientation de l'élève

Question générale: Comment se sont développées tes aspirations professionnelles (profession rêvée, métiers envisagés, etc.) depuis le dernier entretien?

\section{Relances et questions spécifiques}

- Quels sont tes projets aujourd'hui en ce qui a trait à ta formation et à ta profession futures?

- Dans quelle mesure es-tu satisfaite de ton orientation vers ta formation actuelle?

$-\ldots$ 